\title{
Aggregation of dipolar colloidal particles: Geometric effects
}

\author{
Jonathan L. Bentz* \\ Department of Chemistry and Ames Laboratory, Iowa State University, Ames, Iowa 50011, USA \\ John J. Kozak ${ }^{\dagger}$ \\ Beckman Institute, California Institute of Technology, Pasadena, California 91125-7400, USA
}

(Received 15 September 2005; published 31 January 2006)

\begin{abstract}
To understand the importance of confinement and the influence of translational degrees of freedom on aggregation of dipolar colloidal particles, we calculate numerically-exact values for the mean encounter time for two nonspherically symmetric molecules to form a two-molecule cluster, regarded here as a precursor to aggregation. A lattice model is formulated in which the asymmetry of the molecules is accounted for by representing each as a "dimer" in the sense that each molecule is specified to occupy two adjacent lattice sites. The two dimers undergo simultaneous translation, and the mean times for their encounter are determined. Exact numerical results are obtained via application of the theory of finite Markov processes. The results allow one to examine in a detailed way the interplay among such factors as geometrical confinement, system size, translational motion, and specific orientational effects in influencing the aggregation event. The results are compared with previously reported theoretical predictions and experiments on the behavior of dipolar colloidal particles in the presence of an applied magnetic field.
\end{abstract}

DOI: 10.1103/PhysRevE.73.011414

PACS number(s): 61.43.Hv, 83.80.Gv, 02.50.Ga

\section{INTRODUCTION}

This study was undertaken to explore aspects of a recent suggestion of Miguel and Pastor-Satorras [1] that the kinetic growth of field-oriented chains in a dipolar colloidal solution, found experimentally by Melle, Rubio, and Fuller [2] to be characterized by an asymptotic power law for the mean cluster size $S(t)$, is a consequence of the anisotropic diffusion of rodlike objects. In particular, they proposed that hydrodynamic interactions cause the anisotropic diffusion of rods along and normal to their axes, thereby inducing a logarithmic correction in the dependence of the diffusion coefficient on cluster size. Moreover, they confirmed the prediction of Miyazima, Meakin, and Family [3] on the existence of two different aggregation regimes, specifically $d=3$ and $d=1$ (like) behavior, a prediction which was confirmed experimentally [2].

The approach taken here is based on a lattice model, one in which rods, represented by dimers, undergo simultaneous displacements and interact upon first encounter. We mobilize a recently introduced Markovian theory [4-9] which allows one to calculate numerically exact values for the first passage time (or mean walk length) before reaction (or "trapping"). This theory has been applied to a variety of problems, including protein nucleation (see Sec. II). In brief, then, this work differs from that reported in Ref. [1] which was based on Monte Carlo simulations of rods modeled as structureless particles (monomers). That said, we shall find that several of the conclusions reached by Miguel and Pastor-Satorras are

\footnotetext{
*Electronic address: jnbntz@iastate.edu

†Permanent address: DePaul University, 243 South Wabash Avenue, Chicago, IL 60604-2301. Electronic address: kozak@depaul.edu
}

supported by the calculations reported here and, further, that interesting insights are gained by characterizing in detail the configurational (pairwise) interactions of dimeric species.

\section{DESCRIPTION OF THE MODEL}

Throughout this work we are concerned with the seminal aggregation event, an irreversible reaction in which two, simultaneously diffusing asymmetric molecules (hereafter specified as dimers) of the same species $A$ give rise to a cluster $A_{2}$, whereupon the process terminates:

$$
A+A \rightarrow A_{2} .
$$

To study this process we develop a lattice model in which each dimer occupies two lattice sites. Using the theory of finite Markov processes, we explore orientation effects, isotropic and anisotropic diffusion, and the influence of confinement and other finite system size effects on the efficiency of the diffusion-reaction event, Eq. (1).

For definiteness, consider a square-planar lattice subject to periodic boundary conditions in the horizontal direction(s) and confining boundary conditions in the vertical direction(s). Periodic boundary conditions guarantee translational invariance in the horizontal direction, and confining boundary conditions in this context simply define a hard barrier. That is, if the dimer attempts to exit the lattice through this hard barrier, it is reset at the position it occupied immediately prior to its attempt to exit the lattice. Thus the two multipolar molecules experience freedom to move within the fluid in the lateral directions (as defined above), but may be constrained in the vertical direction by exterior confinement of the fluid. It is in this sense that the imposition of upper and lower confining boundaries models the restricted motion of the particles in the $z$ direction.

To account, in part, for the presence of an external field, the dimers are not allowed to rotate, but remain oriented 


\begin{tabular}{|c|c|c|c|c|}
\hline 1 & 2 & 3 & 4 & 5 \\
\hline 6 & 7 & 8 & 9 & 10 \\
\hline 11 & 12 & 13 & 14 & 15 \\
\hline 16 & 17 & 18 & 19 & 20 \\
\hline 21 & 22 & 23 & 24 & 25 \\
\hline
\end{tabular}

FIG. 1. Symmetric $5 \times 5$ square-planar lattice. The left and right boundaries are subject to periodic boundary conditions, while the top and bottom boundaries are confining (see text).

perpendicular to the confining boundaries. As noted above, each dimer occupies two lattice positions in the same column. This is illustrated in Fig. 1 for a $5 \times 5$ lattice, e.g., position $(1,6)$ and position $(13,18)$ specify the coordinates of one, mutual configuration of the two dimers. The dimers are allowed to translate in one of four directions in each time step; to account for isotropic versus anisotropic diffusion events, we explore situations where a dimer can make, respectively, one or two vertical displacements for every one lateral displacement. Again, the process terminates (irreversibly) when two dimers collide.

It is in characterizing the variety of collision events that can occur that the model developed here yields the most detailed insights. Because each dimer occupies two lattice positions, there are two distinct ways for a collision event to occur between two dimers (again recalling that rotational degrees of freedom have been suppressed). First, "two point" collisions are those where two dimers, moving synchronously, attempt to occupy the same two lattice sites; for example, if one dimer is localized initially at position $(7,12)$ on the lattice diagrammed in Fig. 1, and the other is located at position $(9,14)$, there is an interaction configuration whereby, in the next simultaneous step, both dimers attempt to occupy position $(8,13)$.

"One point" collisions are those in which two dimers, again moving synchronously, are in contention at a single lattice site in their next (joint) displacement; for example, if one dimer is located at position $(7,12)$ and the other is located at position $(14,19)$, there is a possible, subsequent (simultaneous) displacement wherein one dimer will attempt to occupy position $(8,13)$, and the second, position $(13,18)$.

The above scenarios can be elaborated further by considering that two dimers, diffusing simultaneously and independently, undergo both translations and rotations on the underlying lattice; for the case of lattices subject to periodic boundary conditions, this generalization was considered in a model of protein nucleation [6]. One can also relax the assumption that the two diffusing species migrate simultaneously. The consequences of assuming asynchronous motions of two species migrating on lattices subject to periodic boundary conditions was explored for the case of structureless particles (monomers); there one finds that the efficiency of the reactive event is influenced significantly not only by the dimensionality of the reaction space, but also the parity
TABLE I. Mean walk length values for dimers on a square planar lattice where $N$ is the linear size. One-point and two-point numbers are reported as percent of total collisions. The last column reports the total number of collisional configurations possible as obtained from the numerically exact solution of the stochastic master equation.

\begin{tabular}{rcccc}
\hline \hline$N$ & $\langle n\rangle$ & One-point $(\%)$ & Two-point $(\%)$ & Collisions \\
\hline 4 & 5.4595 & 77.78 & 22.22 & 180 \\
5 & 10.094 & 81.54 & 18.46 & 325 \\
6 & 16.680 & 83.53 & 16.47 & 510 \\
7 & 25.262 & 84.76 & 15.24 & 735 \\
8 & 36.068 & 85.60 & 14.40 & 1000 \\
9 & 49.022 & 86.21 & 13.79 & 1305 \\
10 & 64.439 & 86.67 & 13.33 & 1650 \\
11 & 82.100 & 87.03 & 12.97 & 2035 \\
12 & 102.43 & 87.32 & 12.68 & 2460 \\
13 & 125.07 & 87.56 & 12.44 & 2925 \\
14 & 150.57 & 87.76 & 12.24 & 3430 \\
15 & 178.41 & 87.92 & 12.08 & 3975 \\
16 & 209.28 & 88.07 & 11.93 & 4560 \\
17 & 242.50 & 88.20 & 11.80 & 5185 \\
18 & 278.93 & 88.31 & 11.69 & 5850 \\
19 & 317.70 & 88.41 & 11.59 & 6555 \\
\hline \hline
\end{tabular}

of the lattice $[7,8]$. These generalizations will not be explored here. Rather, given the physical problem under study, our objective is to explore one-point and two-point collisional events, document the consequences of assuming isotropic versus anisotropic diffusion, and quantify whether (or not) confinement effects can lead to a crossover in the dimensionality of the diffusion-reaction process.

\section{MARKOVIAN FORMULATION OF THE ISOTROPIC CASE}

In the theory of finite Markov processes, one constructs the transition matrix $\mathbf{Q}$, and then determines the fundamental matrix $\mathbf{N}$ of the theory $[10,11]$ via the relation

$$
\mathbf{N}=(\mathbf{I}-\mathbf{Q})^{-1},
$$

where $\mathbf{I}$ is the identity matrix. The row sums of the fundamental matrix are the configuration specific walk lengths, and these are summed to yield the overall mean walk length. In the long-time limit, the reciprocal of the mean walk length $\langle n\rangle$ is asymptotically equal to the smallest eigenvalue of the associated stochastic master equation; thus $\langle n\rangle$ is a measure of the long-time behavior of the diffusion-reaction system.

We consider first the case of isotropic diffusion of the two dimers. Presented in Table I are the numerically exact values for the mean number of simultaneous (synchronous) lattice displacements (i.e., the mean walk length $\langle n\rangle$ ) taken by two dimers on (a sequence of) square planar lattices before collision (either one point or two point) occurs. Since we consider here irreversible aggregation, the process terminates upon first encounter of the two dimers. 

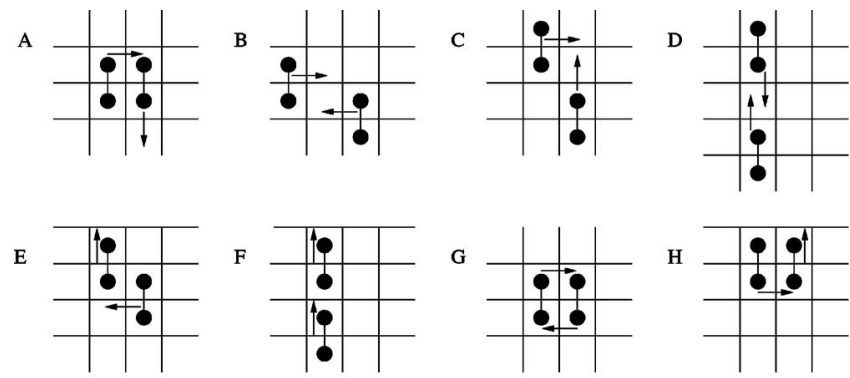

I
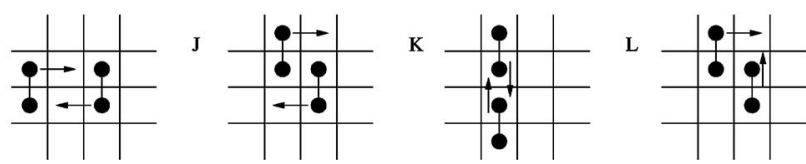

FIG. 2. The 12 symmetry-distinct configurations and relative motions which lead to dimer collision and subsequent aggregation.

An advantage of the Markovian formulation is that one can quantify, for each initial configuration, the firstencounter time (as scaled by $\langle n\rangle$ ) for one-point and two-point collisions. These data are given in Table I; specifically, we report both the total number of collisions and the number of one-point and two-point collisions (the latter given as a percent of the total number).

The symmetry-distinct configurations and relative motions which lead to dimer collisions and subsequent aggregation are diagrammed in Fig. 2. There are 12 symmetrydistinct collisional configurations accessible to the two dimers (see the Appendix for a detailed description of how the symmetry-distinct configurations are categorized). Of these 12 configurations, 3 are two-point collisions and 9 are one-point collisions. This suggests a ratio of two-point to one-point collisions of $1 / 3$. However, in the calculation of the mean walk length via the theory of finite Markov processes, each collisional interaction must be weighted by the number of times it occurs (for each lattice studied). In particular, the finite boundaries of the system, as modeled here by imposing confining boundary conditions on the upper and lower boundaries of the lattice, influence the frequency of occurrence of each type of collision.

The documentation of collision frequency, the type of collision (one-point or two-point), and the concomitant influence of the system boundaries on dimer-dimer collisions is given in Table II. If one uses $x$ and $y$ to designate the linear distance (metric) of a given square-planar lattice in the $x$ and $y$ directions, the following expressions can be developed (by summing the respective one- and two-point frequency ex-
TABLE II. Collision characteristics (frequency of occurrence and boundary dependence) corresponding to the symmetry-distinct configurations diagrammed in Fig. 2. The $x$ and $y$ denote the linear extent of the underlying lattice in the $x$ and $y$ directions.

\begin{tabular}{lccc}
\hline \hline Configuration & Collision & $\begin{array}{c}\text { Frequency } \\
\text { of occurrence }\end{array}$ & $\begin{array}{c}\text { Boundary } \\
\text { dependence }\end{array}$ \\
\hline A & One point & $4 x y-8 x$ & No \\
B & One point & $2 x y-4 x$ & No \\
C & One point & $4 x y-12 x$ & No \\
D & One point & $x y-4 x$ & No \\
E & One point & $4 x$ & Yes \\
F & One point & $2 x$ & Yes \\
G & Two point & $x y-x$ & No \\
H & Two point & $4 x$ & Yes \\
I & Two point & $x y-x$ & No \\
J & One point & $2 x y-4 x$ & No \\
K & One point & $x y-3 x$ & No \\
L & One point & $4 x y-8 x$ & No \\
\hline \hline
\end{tabular}

pressions from Table II) to represent the sum total of the one-point and two-point collisions:

$$
\begin{aligned}
& \text { number of one point collisions }=18 x y-37 x, \\
& \text { number of two point collisions }=2 x y+2 x
\end{aligned}
$$

Consider now the large-system behavior. For the symmetriclattice case $(x=y)$, the data presented in Fig. 2 and Table II suggest that the ratio of two-point to one-point collisions in the isotropic case should be 1/9. As will be seen from the data presented in Table I, this is indeed confirmed. Intuitively, one anticipates that the relative importance of onepoint collisions should increase with increase in system size. These data document quantitatively the emerging dominance of one-point collisions with increase in system size.

Another effect which can be illustrated quite directly in the isotropic case is the influence on dimer aggregation of increasing the cell thickness while holding constant the overall, accessible reaction space. In a lattice model, this can be investigated by comparing results for $m \times n$ vs $n \times m$ lattices, subject to the constraint that the total number of lattice sites is held constant. Thus, for example, notice that the value of $\langle n\rangle$ given in Table III for the $5 \times 10$ lattice is greater than for the $10 \times 5$ lattice (respectively, 32.615 vs 27.682). In fact,

TABLE III. Mean walk length before aggregation of two dimers diffusing independently, synchronously and isotropically on asymmetri-

\begin{tabular}{|c|c|c|c|c|c|c|c|c|c|c|c|c|c|c|}
\hline$x$ & $y$ & $\langle n\rangle$ & $x$ & $y$ & $\langle n\rangle$ & $x$ & $y$ & $\langle n\rangle$ & $x$ & $y$ & $\langle n\rangle$ & $x$ & $y$ & $\langle n\rangle$ \\
\hline 5 & 5 & 10.094 & 5 & 10 & 32.615 & 5 & 15 & 69.575 & 5 & 20 & 120.66 & 5 & 25 & 185.83 \\
\hline 10 & 5 & 27.682 & 10 & 10 & 64.439 & 10 & 15 & 117.34 & 10 & 20 & 185.06 & 10 & 25 & 267.23 \\
\hline 15 & 5 & 53.736 & 15 & 10 & 106.83 & 15 & 15 & 178.41 & 15 & 20 & 265.39 & 15 & 25 & 367.19 \\
\hline 20 & 5 & 88.134 & 20 & 10 & 158.33 & 20 & 15 & 250.79 & & & & & & \\
\hline 25 & 5 & 130.87 & 25 & 10 & 218.35 & 25 & 15 & 332.54 & & & & & & \\
\hline
\end{tabular}
cal lattices. 
one finds quite generally that increasing the spacing of the confining boundaries results in a greater increase in the value of $\langle n\rangle$ than a corresponding increase in the lateral extent of the reaction space. Thus relaxing the geometric constraints imposed on the diffusing dimers by the upper and lower confining boundaries alters significantly the efficiency of the aggregation event.

\section{COMPARISON OF ISOTROPIC VERSUS ANISOTROPIC DIFFUSION}

We now take up the interesting suggestion made by Miguel and Pastor-Satorras [1] regarding the interpretation of the anomalous dynamic exponent $z$ [where the mean cluster size has a power law behavior of $\left.S(t) \sim t^{z}\right]$. These authors focus on a time $T$, the characteristic time for a single aggregation event; in our model, this is the time for two clusters to diffuse, collide, and thus aggregate. The expression they develop for low-dimensional systems is

$$
T \propto \frac{1}{\phi^{2 / d}} \frac{S^{(2+d) / d}}{\ln (S)},
$$

where $\phi$ is the initial volume fraction of dipoles, $d$ is the dimensionality of the system, and $S$ is the average length of the cluster.

The incorporation of a logarithmic correction in Eq. (5) is a consequence of assuming that hydrodynamic interactions cause anisotropic diffusion of the clusters (rods) along versus normal to their axes. To bring our lattice model into correspondence with these ideas, note that the volume fraction here is given by $\phi=4 / x y$, this because two dimers occupy four sites on a lattice defined by a total number $x y$ of sites. Setting $d=2$ and $S=2$ gives

$$
T \propto \frac{x y}{\ln (2)} .
$$

This result stands in contrast to the expression obtained for $T$ assuming that the diffusion is isotropic, i.e., that the motion is not only synchronous, but that displacements in the horizontal and vertical directions are equal. In this case,

$$
T \propto x y .
$$

Thus the qualitative prediction which follows from assuming hydrodynamic interactions is that the time $T$ should decrease. In the theory of finite Markov processes, the characteristic time for the aggregation event is directly proportional to the mean walk length. Thus introducing anisotropic diffusion in the lattice model developed here should lead to a decrease in the mean walk length, all other variables being held constant.

To explore this, suppose we continue to define isotropic diffusion as described above, but now define anisotropic diffusion as a synchronous displacement of two dimers such that displacement in the vertical direction (for each) is characterized by two steps (i.e., two lattice spacings), whereas displacement in the horizontal direction remains a single (lattice) step.

Displayed in Figs. 3-5 are the results obtained comparing the isotropic and anisotropic cases. As noted, these figures

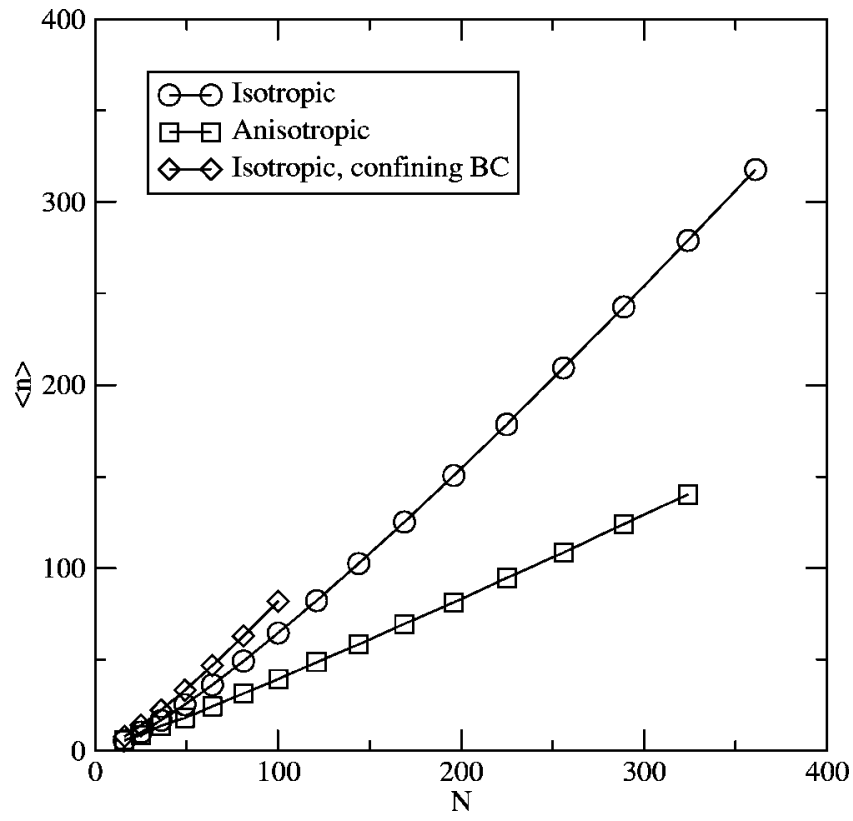

FIG. 3. Mean walk length versus the total number $N$ of lattice sites for dimers undergoing both one-point and two-point collisions on a symmetric square planar lattice. The circles and squares label curves for which confining boundary conditions have been imposed on the upper and lower boundaries, and periodic boundary conditions on the lateral boundaries; the diamonds code lattices subject to confining boundary conditions on all boundaries. In this figure, isotropic and anisotropic refer, respectively, to one-step and two-step vertical displacements of each dimer; horizontal displacements are all unit lattice steps (see text).

give, respectively, the behavior found assuming all one-point and two-point collisions, all one-point collisions (only), and all one-point, vertical (i.e., "head-to-tail") collisions only. As is evident from these figures, introducing anisotropic diffusion (as specified above) leads to a significant decrease in the values calculated for $\langle n\rangle$, and hence is consistent with the prediction that the time scale of the irreversible aggregation event should decrease.

\section{CROSSOVER TO QUASI-ONE-DIMENSIONAL DIFFUSION}

In this section, we focus on the concentration effect noted by Miguel and Pastor-Satorras [1], viz., that there occurs a crossover in the effective dimensionality of the diffusionreaction space from $d \sim 3$ to $d \sim 1$ when the concentration of dipolar particles increases. One way of exploring this idea within the framework of the lattice model developed here is to impose confining boundary conditions, not only on the horizontal boundaries of the system (as implemented in earlier sections), but on the vertical boundaries as well. The basic idea is that an increase in the rod concentration will confine the lateral motion of a given pair of dimers, providing an effective confinement of the system. The question to be addressed, then, is whether the efficiency of the diffusionreaction process is compromised or enhanced with confinement. 


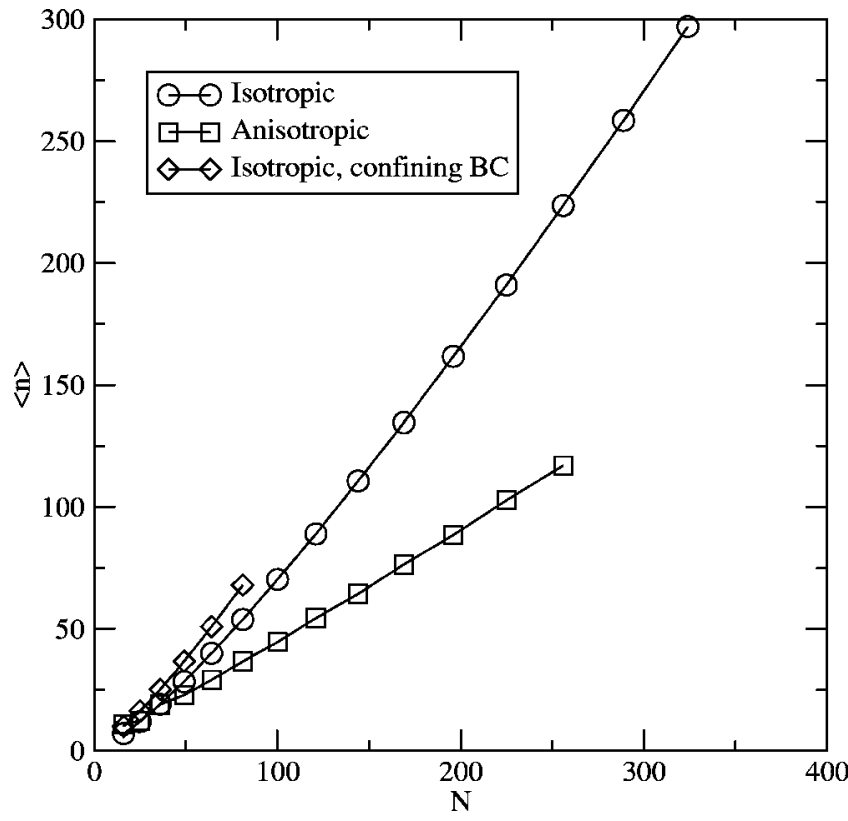

FIG. 4. Mean walk length versus the total number $N$ of lattice sites for dimers undergoing both one-point collisions (only) on a symmetric square planar lattice. The circles and squares label curves for which confining boundary conditions have been imposed on the upper and lower boundaries, and periodic boundary conditions on the lateral boundaries; the diamonds code lattices subject to confining boundary conditions on all boundaries. In this figure, isotropic and anisotropic refer, respectively, to one-step and two-step vertical displacements of each dimer; horizontal displacements are all unit lattice steps (see text).

Before proceeding, it is of interest to recall the seminal work of Montroll and Weiss $[12,13]$ who studied the role of dimensionality in influencing the mean walk length of a monomer migrating on a $d$-dimensional Euclidean lattice in the presence of a deep trap. More precisely, they considered finite lattices subject to periodic boundary conditions. There is one situation where their results have relevance to the case of finite lattices subject to confining boundary conditions, viz., when the deep trap is at the centrosymmetric site of an odd lattice. In this single case, the results of imposing periodic and confining boundary conditions on such a lattice are the same. Montroll and Weiss showed that

$$
\langle n\rangle \sim \begin{cases}N^{2} & \text { in } d=1, \\ N \ln N & \text { in } d=2, \\ N & \text { in } d=3 .\end{cases}
$$

Clearly, for fixed $N$, there is an effective decrease in the efficiency of the diffusion-reaction event as one constrains the diffusive motion of the monomer from $d=3$ to $d=2$ to $d=1$. Within this context, then, the question here is whether one finds a change in the effective dimensionality of the stochastic event as one increases the dimer concentration, i.e., as one constricts the mobility of the two dimers by imposing confining conditions on the lateral boundaries of the system.

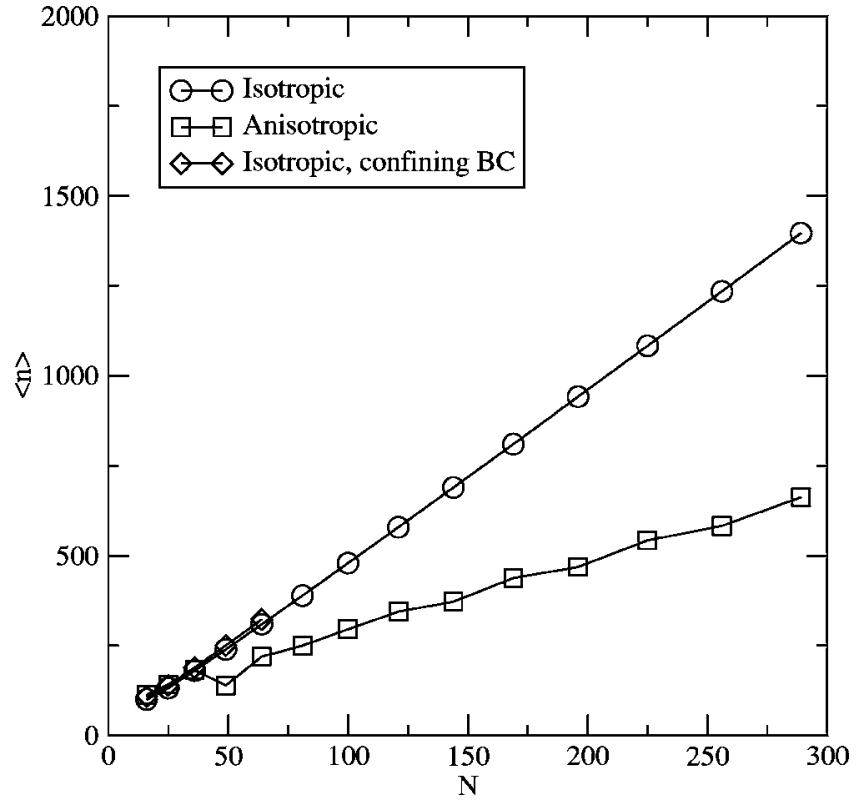

FIG. 5. Mean walk length versus the total number $N$ of lattice sites for dimers undergoing vertical (head-to-tail) collisions (only) on a symmetric square planar lattice. The circles and squares label curves for which confining boundary conditions have been imposed on the upper and lower boundaries, and periodic boundary conditions on the lateral boundaries; the diamonds code lattices subject to confining boundary conditions on all boundaries. In this figure, isotropic and anisotropic refer, respectively, to one-step and two-step vertical displacements of each dimer; horizontal displacements are all unit lattice steps (see text).

To explore this, a series of planar square lattices (up to the $10 \times 10$ ) was considered, and the results (also) displayed in Figs. 3-5 for the case where one-point collisions were assumed. Results obtained for the even more extreme case of asymmetric lattices subject to confining boundary conditions in both the $x$ and $y$ directions are then presented in Fig. 6.

The principal conclusion that can be drawn from the results presented in Figs. 3-5 is that the mean walk length (or, effectively, the mean reaction time) increases with system confinement. Thus constraining the diffusional motion of the dimers by imposing confining boundary conditions effectively contracts the dimensionality of the system (for fixed $N$ ), a result which is consistent with the idea of a crossover in dimensionality when the effective concentration of dipolar particles increases. It is almost certain that the above analysis, guided by the Montroll-Weiss results, Eqs. (8), will fail (or, at best, be incomplete) in more general situations. For example, consider the case where the cell is confined to a fixed width in the $x$ direction, while elongated in the $y$ direction. In particular, in Fig. 6 we display the results of fixing the width of the cell to be three lattice spacings, while increasing the height of the cell as $N / 3$. Although one anticipates that head-to-tail collisions should be far less frequent than all one-point collisions as the system becomes more and more "one dimensional," the curves generated are not only well separated, but considering all lateral collisions, the data show an increase in the reaction efficiency by a factor $\sim 5$. 


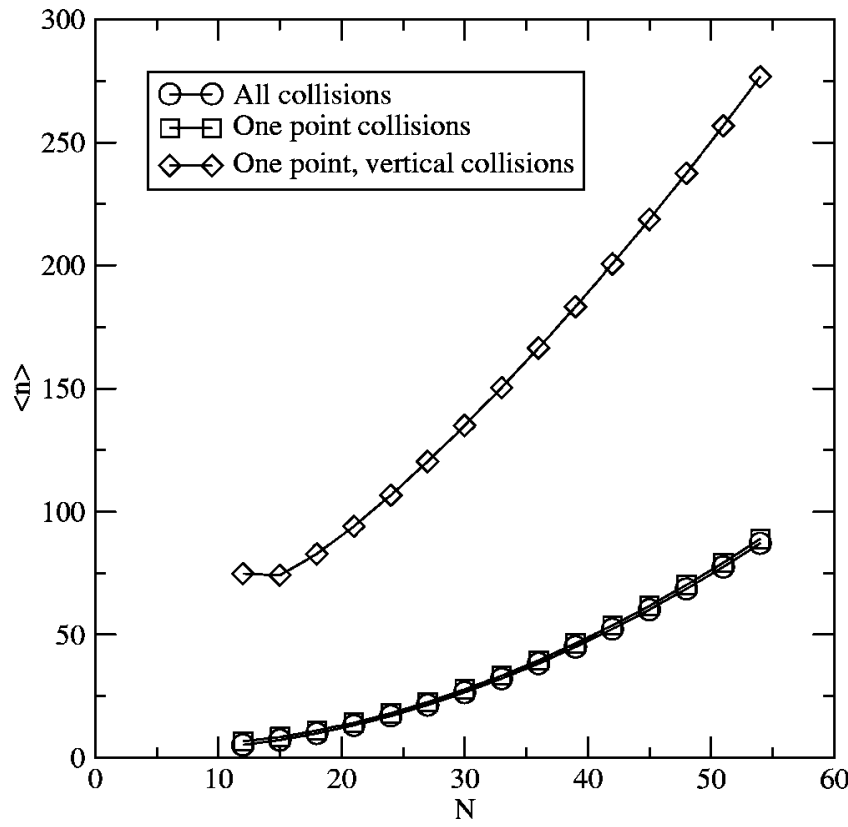

FIG. 6. Mean walk length versus the total number $N$ of lattice sites for asymmetric lattices subject to confining boundary conditions along all boundaries. The three cases considered display the behavior when the linear dimension along the $x$ direction is fixed at $l=3$ and the linear dimension along the $y$ direction is set at $N / 3$.

\section{CONCLUSIONS}

Among the factors which may affect the aggregation of confined dimers, both the restricted diffusion space within which dimers move and specific orientational effects in the seminal collision event are likely to play a role. The calculations reported in this study shed light on the importance and interplay of these two factors.

The Markov method allows one to model interactions occurring at the molecular level, i.e., the joint behavior of two simultaneously translating and interacting dimers. Section III presents numerically exact results for the mean reaction time (as monitored by the mean walk length) of two dimers as a function of system (lattice) size. Though one anticipates that the mean walk length should increase with increase in system size, the quantitative results allow some specific conclusions to be drawn.

First, it was noted that collisional events could be separated into one- and two-point collisions, and quantified by exact expressions. This characterization is a direct consequence of presenting the particle as a structured (dimer) as opposed to an unstructured (monomer) species. This result led to a quantification of the emerging dominance of onepoint collisions (over two-point collisions) with increase in system size. It also allowed us to directly consider collisions shown to be of experimental significance $[2,14,15]$, those for which, as a consequence of an applied field, the majority of the interactions are head-to-tail.

Second, the choice of boundary conditions (confining on top and bottom and periodic on the sides), meant to simulate the features of a partially confined system, was shown to exhibit strikingly different behavior from earlier work [6] where all boundaries were periodic. In particular, a lattice parity effect, which is found when all boundaries are subject to periodic boundary conditions, is not present in this study, where confining boundary conditions were imposed on two (and four) boundaries.

Mean walk length results were reported for the case where $x \neq y$, that is, where the lattices are not symmetric. These data show that increasing the $y$ value of the lattice results in a greater increase in $\langle n\rangle$ than a corresponding increase in the $x$ value of the lattice. Again, this behavior occurs because of geometrical constraints experienced by the diffusing dimers when confronting the (here) upper and lower (confining) boundaries of the reaction system.

Consideration of isotropic versus anisotropic diffusion in the vertical direction (parallel to the assumed field) was incorporated in the model by increasing by a factor of 2 dimer displacements in the vertical directions, while maintaining single lattice displacements in the horizontal direction. Here it was shown that the inclusion of anisotropic diffusion parallel to the field (and the principal axis of the dimers) decreased the value of the mean walk length $\langle n\rangle$, i.e., increased the likelihood of aggregation.

Finally, the examination of quasi-one-dimensional behavior shown in Refs. [1,3] was modeled via the inclusion of confining boundary conditions coupled with an asymmetric elongation of the lattice in the vertical direction. This set of lattice geometries simulates the formation of the quasi-onedimensional regime at high concentrations (which appears due to the aggregates' sizes being of the order of the entire length of the reaction vessel). Results from these calculations showed that the relevance of two-point collisions is almost negligible, as the two lower curves in Fig. 6 are almost identical. This is unsurprising when one considers the onedimensional regime modeled. However, restricting the aggregation events to only vertical collisions increases the mean walk length $\langle n\rangle$ quite dramatically. This shows that while the system is becoming more one-dimensional, the influence of lateral collisions is still important.

The results reported in this work are entirely consistent with those shown previously [1-3], both theoretically and experimentally, regarding the importance of anisotropic diffusion and the onset of quasi-one-dimensional behavior. The results (and calculations) presented allow one to investigate the effect of boundary conditions and system size on the efficiency of the aggregation event. Given the concentration levels of dimer in the system, the influence of these geometrical effects on the efficiency of the reactive event are clearly of seminal importance.

\section{ACKNOWLEDGMENT}

The authors wish to thank Gregoire Nicolis for his helpful comments and suggestions during the preparation of this manuscript.

\section{APPENDIX}

In our formulation of the model there are two classes of joint motions which lead to collision (and thus aggregation). 
These classes are categorized as same-site occupation (SSO) and nearest-neighbor collision (NNC). Consider Fig. 2 and in particular configuration I. When the two dimers execute their respective displacements (which lead to collision) and one examines their positions before and after their displacements, one notices that after their displacements they are both attempting to occupy the same pair of sites. This is the type of collision we call SSO because of the dimers' contention for the same sites on the lattice. Now consider configuration G. Because we have restricted our discussion to dimension $d$ $=2$, position exchange is forbidden since the joint displacement of two dimers does not allow for two dimers to "cross paths" without colliding. This mechanism of collision is called NNE because the dimers started out as nearestneighbors and attempted to exchange positions.

Two-point collisions are defined in this study as only those for which the two dimers are vertically oriented and "lined up" horizontally. Using this criterion, G and I are twopoint collisions. Because of the confining boundary conditions (note the solid line at the top), $\mathrm{H}$ is also a two-point collision; given that we consider here only synchronous collisional events, a first dimer colliding with the boundary is reset thus confronting a second dimer undergoing a horizontal displacement.

All remaining configurations lead to one-point collisions. A, B, C, D, E, and F are all in contention for one lattice site after displacement and thus fall under the SSO class of collisions. $\mathrm{J}$ and $\mathrm{K}$ are exchanging positions, but collide at only one point, thus are NNE collisions. In this picture, case L, although at first sight somewhat more ambiguous, is clearly a one-point collision.
[1] M. Carmen Miguel and R. Pastor-Satorras, Phys. Rev. E 59, 826 (1999).

[2] S. Melle, M. A. Rubio, and G. G. Fuller, Phys. Rev. Lett. 87, 115501 (2001).

[3] S. Miyazima, P. Meakin, and F. Family, Phys. Rev. A 36, 1421 (1987).

[4] J. J. Kozak, C. Nicolis, and G. Nicolis, J. Chem. Phys. 113, 8168 (2000).

[5] C. Nicolis, J. J. Kozak, and G. Nicolis, J. Chem. Phys. 115, 663 (2001).

[6] J. J. Kozak, V. Basios, and G. Nicolis, Biophys. Chem. 105(23), 495 (2003).

[7] J. L. Bentz, J. J. Kozak, E. Abad, and G. Nicolis, Physica A
326, 55 (2003).

[8] E. Abad, G. Nicolis, J. L. Bentz, and J. J. Kozak, Physica A 326, 69 (2003).

[9] J. L. Bentz, J. J. Kozak, and G. Nicolis, Physica A 353, 73 (2005).

[10] J. G. Kemeny and J. L. Snell, Finite Markov Chains (SpringerVerlag, New York, 1976).

[11] J. J. Kozak, Adv. Chem. Phys. 115, 245 (2000).

[12] E. W. Montroll and G. H. Weiss, J. Math. Phys. 6, 167 (1965).

[13] E. W. Montroll, J. Math. Phys. 10, 753 (1969).

[14] T. Ukai and T. Maekawa, Phys. Rev. E 69, 032501 (2004).

[15] S. Melle, O. G. Calderón, M. A. Rubio, and G. G. Fuller, Phys. Rev. E 68, 041503 (2003). 